\title{
Completing partial transversals of Cayley tables of Abelian groups
}

\author{
Jaromy Kuhl Donald McGinn \\ Department of Mathematics and Statistics \\ University of West Florida \\ Pensacola, FL 32514, U.S.A \\ \{jkuhl, dmcginn\}@uwf.edu
}

\author{
Michael William Schroeder \\ Department of Mathematics \\ Marshall University \\ Huntington, WV 25755, U.S.A \\ schroederm@marshall.edu
}

Submitted: Feb 20, 2020; Accepted: Aug 10, 2021; Published: Sep 24, 2021

(C) The authors. Released under the CC BY-ND license (International 4.0).

\begin{abstract}
In 2003 Grüttmüller proved that if $n \geqslant 3$ is odd, then a partial transversal of the Cayley table of $\mathbb{Z}_{n}$ with length 2 is completable to a transversal. Additionally, he conjectured that a partial transversal of the Cayley table of $\mathbb{Z}_{n}$ with length $k$ is completable to a transversal if and only if $n$ is odd and either $n \in\{k, k+1\}$ or $n \geqslant 3 k-1$. Cavenagh, Hämäläinen, and Nelson (in 2009) showed the conjecture is true when $k=3$ and $n$ is prime. In this paper, we prove Grüttmüller's conjecture for $k=2$ and $k=3$ by establishing a more general result for Cayley tables of Abelian groups of odd order.
\end{abstract}

Mathematics Subject Classifications: 05B15, 20K01

\section{Introduction}

It is well-known that Cayley tables of finite cyclic groups have transversals if and only if their order is odd. In fact, such Cayley tables have many transversals, most recently shown by Eberhard, Manners, and Mrazovic [2], who proved that the number of transversals in a Cayley table of $\mathbb{Z}_{n}$ is on the order of $\left(e^{-1 / 2}+o(1)\right) n !^{2} / n^{n-1}$. With this in mind, it seems plausible that for a positive integer $k$ and a large enough $n$, any partial transversal of length $k$ in a Cayley table of $\mathbb{Z}_{n}$ is contained in a transversal.

For a finite group $G$, let $C(G)$ denote the Cayley table of $G$, and let $n \geqslant 3$ be odd. We say that a partial transversal $P$ of $C(G)$ is completable if there exists a transversal $T \subseteq C(G)$ 
such that $P \subseteq T$; we say that $T$ is a completion of $P$. First observe that partial transversals of $C\left(\mathbb{Z}_{n}\right)$ with length $1, n-1$, or $n$, are completable. In particular, partial transversals of length 1 are completable since $C\left(\mathbb{Z}_{n}\right)$ is decomposable into transversals; note that for Latin squares in general, completing partial transversals of length 1 is not always possible [7]. Grüttmüller proved an analogous result for partial transversals of $C\left(\mathbb{Z}_{n}\right)$ with length 2 , as well as other special cases.

Theorem 1 (Grüttmüller [3]). A partial transversal of $C\left(\mathbb{Z}_{n}\right)$ with length 2 is completable if and only if $n$ is odd and $n \geqslant 3$.

Theorem 2 (Grüttmüller [4]). Let $2 \leqslant k \leqslant 7$. Every partial transversal of $C\left(\mathbb{Z}_{n}\right)$ with length $k$, order $n$ odd, and $3 k-1 \leqslant n \leqslant 21$ is completable.

Grüttmüller also established necessary conditions for a partial transversal of $C\left(\mathbb{Z}_{n}\right)$ with length $k$ to be completable.

Theorem 3 (Grüttmüller [4]). If $k \geqslant 3$ and every partial transversal of $C\left(\mathbb{Z}_{n}\right)$ with length $k$ is completable, then $n$ is odd and either $n \in\{k, k+1\}$ or $n \geqslant 3 k-1$.

Stemming from this, Grüttmüller made the following conjecture.

Conjecture 4 (Grüttmüller [4]). If $k \geqslant 1$, then every partial transversal of $C\left(\mathbb{Z}_{n}\right)$ with length $k$ is completable if and only if $n$ is odd and either $n \geqslant 3 k-1$ or $n \in\{k, k+1\}$.

The only known progress on Conjecture 4 is due to Cavenagh, Hämäläinen, and Nelson.

Theorem 5 (Cavenagh et al. [1]). Let $n \geqslant 11$ and $n$ prime. Then any partial transversal of $C\left(\mathbb{Z}_{n}\right)$ with length 3 is completable.

In this paper we settle Grüttmüller's conjecture for the case $k=3$. In fact, we prove the following stronger result.

Theorem 6. For an Abelian group $G$ of odd order $n$ and $k \in\{2,3\}$, every partial transversal of $C(G)$ with length $k$ is completable if and only if either $n \geqslant 3 k-1$ or $n \in\{k, k+1\}$.

Observe that for any Abelian group $G$ of odd order $n$, a partial transversal of $C(G)$ with length $1, n-1$, or $n$ is completable for the same reasons as when $G$ is cyclic and thus, for the same reasons, an appropriate first step in this generalization is to establish the conditions under which partial transversals of $C(G)$ with lengths 2 and 3 are completable.

Lastly, we mention here that the work cited above was presented in terms of diagonally cyclic Latin squares. For odd $n$, a Latin square $L \subseteq \mathbb{Z}_{n} \times \mathbb{Z}_{n} \times \mathbb{Z}_{n}$ is diagonally cyclic if $(i+1, j+1, k+1) \in L$ whenever $(i, j, k) \in L$. There is a one-to-one correspondence between diagonally cyclic Latin squares of order $n$ and transversals in $C\left(\mathbb{Z}_{n}\right)$ as shown in the following example. 


\begin{tabular}{|l|l|l|l|l|}
\hline 4 & 2 & 0 & 3 & 1 \\
\hline 2 & 0 & 3 & 1 & 4 \\
\hline 0 & 3 & 1 & 4 & 2 \\
\hline 3 & 1 & 4 & 2 & 0 \\
\hline 1 & 4 & 2 & 0 & 3 \\
\hline
\end{tabular}

$L$

\begin{tabular}{|l|l|l|l|l|}
\hline 0 & 1 & 2 & 3 & 4 \\
\hline 1 & 2 & 3 & 4 & 0 \\
\hline 2 & 3 & 4 & 0 & 1 \\
\hline 3 & 4 & 0 & 1 & 2 \\
\hline 4 & 0 & 1 & 2 & 3 \\
\hline
\end{tabular}

$C\left(\mathbb{Z}_{5}\right)$

Figure 1: A diagonally cyclic Latin square of order 5 and its corresponding transversal of $C\left(\mathbb{Z}_{5}\right)$.

Example 7. Let $L$ be the diagonally cyclic Latin square of order 5 given in Figure 1 . Note that the first row of $L$ determines $L$, and the map which associates $\left(0, i, s_{i}\right) \in L$ with $\left(s_{i}-i, i, s_{i}\right) \in C\left(\mathbb{Z}_{5}\right)$ demonstrates the correspondence between $L$ and the highlighted transversal of $C\left(\mathbb{Z}_{5}\right)$.

Thus completing partial Latin squares of order $n$ to diagonally cyclic Latin squares of order $n$ is equivalent to completing partial transversals of $C\left(\mathbb{Z}_{n}\right)$ to transversals. With this in mind, the theorems and conjecture above can be restated using diagonally cyclic Latin squares. For more on diagonally cyclic Latin squares and related objects, see the survey by Wanless [6].

\section{Coset Blocks and Chains}

Let $n$ be a positive odd integer and $G$ be an Abelian group of order $n$, expressed additively. We treat $C(G)$ as a Latin square, that is, as a subset of $G \times G \times G$, where $(a, b, c) \in C(G)$ if and only if $c=a+b$ in $G$.

Observe that when $k=2$ or $k=3$, Conjecture 4 is true for Abelian groups of prime order since these groups are cyclic. For the remainder of the paper, we focus on developing machinery for handling $C(G)$ when $G$ has composite order.

Let $n$ be composite with prime divisor $q$ and $m$ be defined so that $n=m q$. Define $\ell$ so that $q=2 \ell+1$. Let $H$ be a subgroup of $G$ of order $m$. We use 0 for the zero element of $H$ and for each $k \in \mathbb{Z}$ and for each $g \in G$, let $k g=g+g+\cdots+g$ ( $k$ times). Note that the factor group $G / H$ has prime order $q$ and thus $G / H$ is isomorphic to $\mathbb{Z}_{q}$; let $\mu: \mathbb{Z}_{q} \rightarrow G / H$ be such an isomorphism. Let $H_{0}=H$ and $H_{1}, H_{2}, \ldots, H_{q-1}$ denote the remaining cosets of $H$ in $G / H$, where $H_{i}=\mu(i)$ for each $i \in \mathbb{Z}_{q}$.

Observe that for each $i, j \in \mathbb{Z}_{q},\left\{(a, b, a+b) \in C(G): a \in H_{i}, b \in H_{j}\right\}$ is a Latin square which is a subset of $H_{i} \times H_{j} \times H_{i+j}$ that is isomorphic (as a Latin square) to $C(H)$. We call this Cayley table a coset block of order $m$ in $C(G)$ and we denote it as $C_{i, j}(H ; G, \mu)$ (or when the context makes it unambiguous, simply $C_{i, j}(H)$ ); note that $C_{0,0}(H)=C(H)$. We denote $C_{0, d}(H) \cup C_{1, d+1}(H) \cup \cdots \cup C_{q-1, d+q-1}(H)$ as the $d$ th block diagonal of $C(G)$ with respect to $(H, \mu)$ and call the 0th block diagonal the main block diagonal. 
For a subset $P$ of $C(G)$, we let $P^{r}, P^{c}$, and $P^{s}$ denote the set of rows, columns, and symbols used by the triples in $P$. Additionally for each $i \in \mathbb{Z}_{q}$, let $P_{i}^{r}=P^{r} \cap H_{i}, P_{i}^{c}=P^{c} \cap H_{i}$, and $P_{i}^{s}=P^{s} \cap H_{i}$.

We begin by observing an invariance in $C(G)$; its use will reduce the complexity of later arguments.

Observation 8. Let $a, b \in G$, and $\psi$ be an automorphism of $G$. Then the following are automorphisms of $C(G)$ :

$$
\begin{aligned}
\delta_{a, b}(x, y, z) & =(x+a, y+b, z+a+b), \text { and } \\
\psi(x, y, z) & =(\psi(x), \psi(y), \psi(z)) .
\end{aligned}
$$

We often leverage that if $\phi$ is an automorphism of $C(G)$ and $P$ is a partial transversal of $C(G)$, then $P$ is completable if and only if $\phi(P)$ is completable as well. In particular, we frequently assume that $(0,0,0)$ belongs to $P$. Indeed if $(a, b, a+b) \in P$, then $(0,0,0) \in$ $\delta_{-a,-b}(P)$, and thus we can work with the image of $P$ instead. Furthermore, for an integer $c$ relatively prime to $|G|$, we use that $\xi_{c}: G \rightarrow G$ given by $\xi_{c}(g)=c g$ is an automorphism of $G$, and therefore gives rise to an automorphism of $C(G)$.

The constructions used in this paper hinge heavily on building chains, which are now defined, with examples that follow.

Definition 9. Let $G$ be an Abelian group of odd order $m q$ with $q$ prime, $m \geqslant 3$, and an order $m$ subgroup $H$. Let $\mu: \mathbb{Z}_{q} \rightarrow G / H$ be an isomorphism with $\mu(i)=H_{i}$ for $i \in \mathbb{Z}_{q}$.

Let $1 \leqslant d \leqslant q-1$ and both $\left\{x_{i} \in H_{i}: i \in \mathbb{Z}_{q}\right\}$ and $\left\{y_{i} \in H_{i}: i \in \mathbb{Z}_{q}\right\}$ be systems of distinct representatives (SDRs) of $G / H$. The partial transversal $P=\left\{\left(x_{i}, y_{i}, x_{i}+y_{i}\right): i \in \mathbb{Z}_{q}\right\}$ of $C(G)$ is a $(d, H)$-chain of $C(G)$ with respect to $\mu$ if

$$
x_{i}+y_{i}=x_{i+\ell d}+y_{i+(\ell+1) d} \text { for each } i \in \mathbb{Z}_{q} .
$$

Define $\sigma(P)$, called the swap of $P$, as the subset $\left\{\left(x_{i}, y_{i+d}, x_{i}+y_{i+d}\right): i \in \mathbb{Z}_{q}\right\}$ of $C(G)$. Since $P$ satisfies the conditions outlined in $(1), \sigma(P)$ is also a partial transversal of $C(G)$ such that $P^{r}=\sigma(P)^{r}, P^{c}=\sigma(P)^{c}$, and $P^{s}=\sigma(P)^{s}$. Note that $P$ consists of $q$ cells, each of which are representatives of distinct coset blocks on the main block diagonal of $C(G)$ with respect to $(H, \mu)$, while those in $\sigma(P)$ represent the coset blocks on the $d$ th block diagonal of $C(G)$ with respect to $(H, \mu)$. Furthermore, if $T$ is a completion of $P$ in $C(G)$, then $(T \backslash P) \cup \sigma(P)$ is also a transversal of $C(G)$.

Example 10. Let $G=\mathbb{Z}_{15}, H=\{0,5,10\}, \mu: \mathbb{Z}_{5} \rightarrow G / H$ be the canonical isomorphism, and $d=3$; so $n=15, m=3, q=5$, and $\ell=2$. Then

$$
P=\{(0,5,5),(6,6,12),(7,12,4),(13,8,6),(9,14,8)\}
$$

is a $(3, H)$-chain with respect to $\mu$, as evidenced by the table in Figure 2 , and is illustrated in Figure 3(a). Furthermore $P^{r}=\{0,6,7,13,9\}, P^{c}=\{5,6,12,8,14\}, P^{s}=\{5,6,12,8,4\}$, and

$$
\sigma(P)=\{(0,8,8),(6,14,5),(7,5,12),(13,6,4),(9,12,6)\}
$$




\begin{tabular}{|r|rrrrr|}
\hline$i$ & 0 & 1 & 2 & 3 & 4 \\
\hline$x_{i}$ & 0 & 6 & 7 & 13 & 9 \\
\hline$y_{i}$ & 5 & 6 & 12 & 8 & 14 \\
\hline$x_{i}+y_{i}$ & 5 & 12 & 4 & 6 & 8 \\
\hline$x_{i+\ell d}$ & 6 & 7 & 13 & 9 & 0 \\
\hline$y_{i+(\ell+1) d}$ & 14 & 5 & 6 & 12 & 8 \\
\hline$x_{i+\ell d}+y_{i+(\ell+1) d}$ & 5 & 12 & 4 & 6 & 8 \\
\hline
\end{tabular}

Figure 2: A summary of values corresponding to the construction in Example 10.

\begin{tabular}{|c|c|c|c|c|c|c|c|c|c|c|c|c|c|c|c|}
\hline & & & 10 & & & 11 & & & 12 & 3 & & 13 & 4 & & 14 \\
\hline & 0 & 5 & 10 & 1 & 6 & 11 & 2 & 7 & 12 & 3 & 8 & 13 & 4 & 9 & 14 \\
\hline & 5 & 10 & 0 & 6 & 11 & 1 & 7 & 12 & 2 & 8 & 13 & 3 & 9 & 14 & \\
\hline 0 & 10 & 0 & 5 & 11 & 1 & 6 & 12 & 2 & 7 & 13 & 3 & 8 & 14 & 4 & 9 \\
\hline 1 & 1 & 6 & 11 & 2 & 7 & 12 & 3 & 8 & 13 & 4 & 9 & 14 & 5 & 10 & 0 \\
\hline & 6 & 11 & 1 & 7 & 12 & 2 & 8 & 13 & 3 & 9 & 14 & 4 & 10 & 0 & 5 \\
\hline & 11 & 1 & 6 & 12 & 2 & 7 & 13 & 3 & 8 & 14 & 4 & 9 & 0 & 5 & 10 \\
\hline 2 & 2 & 7 & 12 & 3 & 8 & 13 & 4 & 9 & 14 & 5 & 10 & 0 & 6 & 11 & 1 \\
\hline 7 & 7 & 12 & 2 & 8 & 13 & 3 & 9 & 14 & 4 & 10 & 0 & 5 & 11 & 1 & 6 \\
\hline 12 & 12 & 2 & 7 & 13 & 3 & 8 & 14 & 4 & 9 & 0 & 5 & 10 & 1 & 6 & 11 \\
\hline 3 & 3 & 8 & 13 & 4 & 9 & 14 & 5 & 10 & 0 & 6 & 11 & 1 & 7 & 12 & 2 \\
\hline 8 & 8 & 13 & 3 & 9 & 14 & 4 & 10 & 0 & 5 & 11 & 1 & 6 & 12 & 2 & 7 \\
\hline & 13 & 3 & 8 & 14 & 4 & 9 & 0 & 5 & 10 & 1 & 6 & 11 & 2 & 7 & 12 \\
\hline & 4 & 9 & 14 & 5 & 10 & 0 & 6 & 11 & 1 & 7 & 12 & 2 & 8 & 13 & 3 \\
\hline & 9 & 14 & 4 & 10 & 0 & 5 & 11 & 1 & 6 & 12 & 2 & 7 & 13 & 3 & \\
\hline & 14 & 4 & 9 & 0 & 5 & 10 & 1 & 6 & 11 & 2 & 7 & 12 & 3 & 8 & 13 \\
\hline
\end{tabular}

(a)

\begin{tabular}{|c|c|c|c|c|c|c|c|c|c|c|c|c|c|c|c|}
\hline & & & 10 & & & & & & 1 & & & 1 & 4 & & 14 \\
\hline & 0 & 5 & 10 & 1 & 6 & 11 & 2 & 7 & 12 & 3 & 8 & 13 & 4 & 9 & 14 \\
\hline & 5 & 10 & 0 & 6 & 11 & 1 & 7 & 12 & 2 & 8 & 13 & 3 & 9 & 14 & 4 \\
\hline & 10 & 0 & 5 & 11 & 1 & 6 & 12 & 2 & 7 & 13 & 3 & 8 & 14 & 4 & 9 \\
\hline & 1 & 6 & 11 & 2 & 7 & 12 & 3 & 8 & 13 & 4 & 9 & 14 & 5 & 10 & 0 \\
\hline & 6 & 11 & 1 & 7 & 12 & 2 & 8 & 13 & 3 & 9 & 14 & 4 & 10 & 0 & 5 \\
\hline & 11 & 1 & 6 & 12 & 2 & 7 & 13 & 3 & 8 & 14 & 4 & 9 & 0 & 5 & 10 \\
\hline & 2 & 7 & 12 & 3 & 8 & 13 & 4 & 9 & 14 & 5 & 10 & 0 & 6 & 11 & 1 \\
\hline 7 & 7 & 12 & 2 & 8 & 13 & 3 & 9 & 14 & 4 & 10 & 0 & 5 & 11 & 1 & 6 \\
\hline 2 & 12 & 2 & 7 & 13 & 3 & 8 & 14 & 4 & 9 & 0 & 5 & 10 & 1 & 6 & 11 \\
\hline 3 & 3 & 8 & 13 & 4 & 9 & 14 & 5 & 10 & 0 & 6 & 11 & 1 & 7 & 12 & 2 \\
\hline 8 & 8 & 13 & 3 & 9 & 14 & 4 & 10 & 0 & 5 & 11 & 1 & 6 & 12 & 2 & 7 \\
\hline & 13 & 3 & 8 & 14 & 4 & 9 & 0 & 5 & 10 & 1 & 6 & 11 & 2 & 7 & 12 \\
\hline & 4 & 9 & 14 & 5 & 10 & 0 & 6 & 11 & 1 & 7 & 12 & 2 & 8 & 13 & 3 \\
\hline & 9 & 14 & 4 & 10 & 0 & 5 & 11 & 1 & 6 & 12 & 2 & 7 & 13 & 3 & 8 \\
\hline & 14 & 4 & 9 & 0 & 5 & 10 & 1 & 6 & 11 & 2 & 7 & 12 & 3 & & 13 \\
\hline
\end{tabular}

(b)

Figure 3: (a) The $(3, H)$-chain $P$ and its swap $\sigma(P)$ given in Example 10 and (b) the offset $P_{2}(5)$ and its swap given in Example 13. The cells of the chains are shaded and outlined, while the cells of their swaps are only shaded.

We now present a classification result which highlights how to construct a chain.

Lemma 11. Let $G$ be an Abelian group of odd order $m q$ with $q$ prime, $m \geqslant 3$, and an order $m$ subgroup $H$. Let $\mu: \mathbb{Z}_{q} \rightarrow G / H$ be an isomorphism with $\mu(i)=H_{i}$ for $i \in \mathbb{Z}_{q}$.

Let $1 \leqslant d \leqslant q-1$. A $(d, H)$-chain of $C(G)$ with respect to $\mu$ is determined by an $S D R$ of $G / H$, each being a symbol used by the chain, along with a row used by one cell of the chain.

Proof. Let $S=\left\{s_{i} \in H_{i}: i \in \mathbb{Z}_{q}\right\}$ be an SDR of $G / H, t \in \mathbb{Z}_{q}$, and $x_{t} \in H_{t}$. Define $y_{t}, x_{t-d}, y_{t-d}, \ldots, x_{t-(q-1) d}, y_{t-(q-1) d}$ by the equations

$$
\begin{aligned}
& y_{t-j d}=s_{2 t-2 j d}-x_{t-j d} \text { for each } j \in \mathbb{Z}_{q}, \text { and } \\
& x_{t-j d}=s_{2 t-(2 j-1) d}-y_{t-(j-1) d} \text { for each nonzero } j \in \mathbb{Z}_{q} .
\end{aligned}
$$


These $2 q-1$ equations uniquely determine the partial transversal $P=\left\{\left(x_{j}, y_{j}, s_{2 j}\right): j \in \mathbb{Z}_{q}\right\}$ for which

$$
\begin{aligned}
x_{k}+y_{k} & =s_{2 k} \text { for all } k \in \mathbb{Z}_{q} \text { and } \\
x_{k+\ell d}+y_{k+(\ell+1) d} & =s_{2 k} \text { for all } k \in \mathbb{Z}_{q} \text { with } k \neq t-\ell d .
\end{aligned}
$$

Hence (1) holds when $i \neq t-\ell d$. Thus, to show $P$ is a $(d, H)$-chain of $C(G)$ with respect to $\mu$, we must demonstrate that $x_{t-\ell d+\ell d}+y_{t-\ell d+(\ell+1) d}=s_{2(t-\ell d)}$, or equivalently $x_{t}+y_{t+d}=s_{2 t+d}$. Since $G$ is Abelian, we have that

$$
\begin{aligned}
x_{t} & +y_{t+d} \\
& =x_{t}+s_{2 t+2 d}-x_{t+d} \\
& =x_{t}+s_{2 t+2 d}-s_{2 t+3 d}+y_{t+2 d} \\
& =x_{t}+s_{2 t+2 d}-s_{2 t+3 d}+s_{2 t+4 d}-x_{t+2 d} \\
& =x_{t}+s_{2 t+2 d}-s_{2 t+3 d}+s_{2 t+4 d}-s_{2 t+5 d}+s_{2 t+6 d}-x_{t+3 d} \\
& \vdots \\
& =x_{t}+s_{2 t+2 d}-s_{2 t+3 d}+s_{2 t+4 d}-s_{2 t+5 d}+s_{2 t+6 d}-\cdots-s_{2 t+(2 q-1) d}+s_{2 t+2 q d}-x_{t+q d} \\
& =\sum_{j=2}^{q}(-1)^{j} s_{2 t+j d}+\sum_{j=1}^{q}(-1)^{j+q} s_{2 t+(j+q) d} \\
& =\sum_{j=2}^{q}(-1)^{j} s_{2 t+j d}-\sum_{j=1}^{q}(-1)^{j} s_{2 t+j d} \\
& =s_{2 t+d} .
\end{aligned}
$$

Therefore, $P$ is a $(d, H)$-chain of $C(G)$ with respect to $\mu$.

In later arguments we need disjoint chains - chains whose rows, columns, and symbol sets are disjoint. To that end we make the following observation which allows for the modest manipulation of a chain.

Definition 12. Let $G$ be an Abelian group of odd order $m q$ with $q$ prime, $m \geqslant 3$, and an order $m$ subgroup $H$. Let $\mu: \mathbb{Z}_{q} \rightarrow G / H$ be an isomorphism with $\mu(i)=H_{i}$ for $i \in \mathbb{Z}_{q}$.

Suppose that $P=\left\{\left(x_{j}, y_{j}, x_{j}+y_{j}\right): j \in \mathbb{Z}_{q}, x_{j}, y_{j} \in H_{j}\right\}$ is a $(d, H)$-chain of $C(G)$ with respect to $\mu$. Let $i \in \mathbb{Z}_{q}$ and let $v \in H$. Define $s=i+d(\ell+1)(\bmod q)$. Then the offset $P_{i}(v)$ of $P$ is the partial transversal of $C(G)$ obtained by replacing $\left(x_{i}, y_{i}, x_{i}+y_{i}\right)$ and $\left(x_{s}, y_{s}, x_{s}+y_{s}\right)$ with $\left(x_{i}+v, y_{i}, x_{i}+v+y_{i}\right)$ and $\left(x_{s}, y_{s}+v, x_{s}+y_{s}+v\right)$ in $P$. Note $P_{i}(v)$ is also a $(d, H)$-chain of $C(G)$ with respect to $\mu$.

Example 13. Again let $G=\mathbb{Z}_{15}, H=\{0,5,10\}, \mu: \mathbb{Z}_{5} \rightarrow G / H$ be the canonical isomorphism, and $d=3$; so $n=15, m=3, q=5$, and $\ell=2$. Let $P$ be the $(3, H)$-chain of $C(G)$ with respect to $\mu$ given in Example 10 and $i=2$. So $s=1$ and hence

$$
\begin{aligned}
P & =\{(0,5,5),(6,6,12),(7,12,4),(13,8,6),(9,14,8)\} \text { and } \\
P_{2}(5) & =\{(0,5,5),(6,11,2),(12,12,9),(13,8,6),(9,14,8)\} .
\end{aligned}
$$


Observe that $P_{2}(5)$ is also a $(3, H)$-chain of $C(G)$ with respect to $\mu$. See Figure 3(b).

In practice, to find a completion of a partial transversal $P$ of $C(G)$, we partition $P$ into two parts $P^{\prime}$ and $P^{\prime \prime}$, then find a chain $C$ such that $C \cup P^{\prime}$ is completable and $P^{\prime \prime} \subseteq \sigma(C)$. Thus if $\tau$ is a completion of $C \cup P^{\prime}$, then $(\tau \backslash C) \cup \sigma(C)$ is a completion of $P$ in $C(G)$. To that end we have the following two technical lemmas which allow for the construction of chains subject to certain conditions.

Lemma 14. Let $G$ be an Abelian group of odd order $m q$ with $q$ prime, $m \geqslant 3$, and an order $m$ subgroup $H$. Let $\mu: \mathbb{Z}_{q} \rightarrow G / H$ be an isomorphism with $\mu(i)=H_{i}$ for $i \in \mathbb{Z}_{q}$.

Let $d, i \in \mathbb{Z}_{q}$ with $d \neq 0$. If $x_{i}, y_{i} \in H_{i}$ and $y_{i+d} \in H_{i+d}$, then there is a $(d, H)$-chain of $C(G)$ with respect to $\mu$ which contains $\left(x_{i}, y_{i}, x_{i}+y_{i}\right)$, and whose swap contains $\left(x_{i}, y_{i+d}, x_{i}+y_{i+d}\right)$.

Proof. By Observation 8, we may assume that $i=0$ and $x_{0}=y_{0}=0$. So we must show that a $(d, H)$-chain of $C(G)$ with respect to $\mu$ exists which contains $(0,0,0)$ and whose swap contains $\left(0, y_{d}, y_{d}\right)$.

We begin by letting $\left\{s_{i} \in H_{i}: i \in \mathbb{Z}_{q}\right\}$ be an SDR of $G / H$ for which $s_{0}=0$ and $s_{d}=y_{d}$. Using Lemma 11 , there is a unique $(d, H)$-chain $P=\left\{\left(a_{i}, b_{i}, s_{2 i}\right): i \in \mathbb{Z}_{q}\right\}$ of $C(G)$ with respect to $\mu$ for which $a_{0}=0$. Since $a_{0}+b_{0}=s_{0}$ we have $b_{0}=0$, so $(0,0,0) \in P$. Additionally $\sigma(P)$ must contain $\left(a_{0}, b_{d}, s_{d}\right)=\left(0, b_{d}, y_{d}\right)$. So $0+b_{d}=y_{d}$ and therefore $b_{d}=y_{d}$. Hence $\left(0, y_{d}, y_{d}\right) \in \sigma(P)$.

Lemma 15. Let $G$ be an Abelian group of odd order $m q$ with $q$ prime, $m \geqslant 3$, and an order $m$ subgroup $H$. Let $\mu: \mathbb{Z}_{q} \rightarrow G / H$ be an isomorphism with $\mu(t)=H_{t}$ for $t \in \mathbb{Z}_{q}$. Suppose

- $d, i, j \in \mathbb{Z}_{q}$ such that $d$ is nonzero and $2 i=2 j+d$,

- $w, z \in H_{i}, w^{\prime} \in H_{j}$, and $z^{\prime} \in H_{j+d}$ such that $w+z=w^{\prime}+z^{\prime}$, and

- $X$ is a collection of triples such that $w, w^{\prime} \notin X^{r}, z, z^{\prime} \notin X^{c}$, and $w+z \notin X^{s}$.

Then there exists a $(d, H)$-chain of $C(G)$ with respect to $\mu$ which is row-, column-, and symbol-disjoint from $X$, contains $(w, z, w+z)$, and whose swap contains $\left(w^{\prime}, z^{\prime}, w^{\prime}+z^{\prime}\right)$ if

- $m>2|X|$ or

- $m>\max \left\{\left|X_{t}^{r}\right|: t \in \mathbb{Z}_{q}\right\}+\max \left\{\left|X_{t}^{c}\right|: t \in \mathbb{Z}_{q}\right\}+2 \cdot \max \left\{\left|X_{t}^{s}\right|: t \in \mathbb{Z}_{q}\right\}$.

Proof. By using an appropriate operation from Observation 8, we may assume $i=0$ and $w=z=0$; hence $j=\ell d$ and $z^{\prime}=-w^{\prime}$.

First let $S=\left\{s_{t d} \in H_{t d} \backslash X_{t d}^{s}: t \in\{0,1, \ldots, q-3\}\right\}$ be a partial SDR of $G / H$ for which $s_{0}=0$. Note that if $m>2|X|$, then

$\left|H_{(q-2) d}\right|=m>2|X| \geqslant\left|X_{(q-2) d}^{s}\right|+\left|\left\{a \in H_{(q-2) d}: w^{\prime}+a-s_{(q-3) d}+s_{(q-4) d}-\cdots+s_{d} \in X^{s}\right\}\right|$, 
or if $m>\max \left\{\left|X_{t}^{r}\right|: t \in \mathbb{Z}_{q}\right\}+\max \left\{\left|X_{t}^{c}\right|: t \in \mathbb{Z}_{q}\right\}+2 \cdot \max \left\{\left|X_{t}^{s}\right|: t \in \mathbb{Z}_{q}\right\}$, then

$$
\begin{aligned}
\left|H_{(q-2) d}\right| & =m>2 \cdot \max \left\{\left|X_{t}^{s}\right|: t \in \mathbb{Z}_{q}\right\} \geqslant\left|X_{(q-2) d}^{s}\right|+\left|X_{(q-1) d}^{s}\right| \\
& \geqslant\left|X_{(q-2) d}^{s}\right|+\left|\left\{a \in H_{(q-2) d}: w^{\prime}+a-s_{(q-3) d}+s_{(q-4) d}-\cdots+s_{d} \in X_{(q-1) d}^{s}\right\}\right| .
\end{aligned}
$$

Hence there exists $s_{(q-2) d} \in H_{(q-2) d} \backslash X_{(q-2) d}^{s}$ so that $w^{\prime}+s_{(q-2) d}-s_{(q-3) d}+s_{(q-4) d}-\cdots+s_{d}$ (we denote this sum as $s_{(q-1) d}$ ) belongs to $H_{(q-1) d} \backslash X_{(q-1) d}^{s}$. Therefore $S \cup\left\{s_{(q-2) d}, s_{(q-1) d}\right\}$ is an SDR of $G / H$ which is disjoint from $X^{s}$.

Let $P=\left\{\left(a_{t}, b_{t}, a_{t}+b_{t}\right) \in C_{t, t}(H): t \in \mathbb{Z}_{q}\right\}$ be the $(d, H)$-chain of $C(G)$ with respect to $\mu$ using row 0 and symbols in $S \cup\left\{s_{(q-2) d}, s_{(q-1) d}\right\}$. Then $(0,0,0) \in P$ and, by using the equations in (2), we have that $a_{\ell d}=s_{(q-1) d}-s_{(q-2) d}+\cdots+s_{2 d}-s_{d}$; therefore $a_{\ell d}=w^{\prime}$. Since $b_{(\ell+1) d}+a_{\ell d}=s_{0}=0$, we have that $b_{(\ell+1) d}=z^{\prime}$, and hence $\left(w^{\prime}, z^{\prime}, 0\right) \in \sigma(P)$.

At this point, $P$ and $X$ may not be disjoint in rows and columns. In what follows, we use offsets to make $P$ disjoint from $X$ while maintaining that $(0,0,0) \in P$ and $\left(w^{\prime}, z^{\prime}, 0\right) \in \sigma(P)$. Suppose that, for some $t \in \mathbb{Z}_{q}$, either $a_{t} \in X^{r}$ or $b_{t+(\ell+1) d} \in X^{c}$. Note that $t \neq 0$ and $t \neq \ell d$, as $\left\{(0,0,0),\left(w^{\prime}, z^{\prime}, 0\right)\right\}$ is disjoint from $X$. Consider the sets

$$
\begin{aligned}
& X_{1}=\left\{(a, b, c) \in X: a=a_{t}+v \text { for some } v \in H\right\}, \\
& X_{2}=\left\{(a, b, c) \in X: b=b_{t+(\ell+1) d}+v \text { for some } v \in H\right\}, \\
& X_{3}=\left\{(a, b, c) \in X: c=a_{t}+b_{t}+v \text { for some } v \in H\right\}, \text { and } \\
& X_{4}=\left\{(a, b, c) \in X: c=a_{t+(\ell+1) d}+b_{t+(\ell+1) d}+v \text { for some } v \in H\right\} .
\end{aligned}
$$

Note that if $(a, b, c) \in X_{1}$, then $a \in H_{t}$; if $(a, b, c) \in X_{2}$, then $b \in H_{t+(\ell+1) d}$; if $(a, b, c) \in X_{3}$, then $c \in H_{2 t}$; and if $(a, b, c) \in X_{4}$, then $c \in H_{2 t+d}$. It follows that any triple in $X$ belongs to at most two subsets $X_{t}, t \in\{1,2,3,4\}$. Therefore $\left|X_{1}\right|+\left|X_{2}\right|+\left|X_{3}\right|+\left|X_{4}\right| \leqslant 2|X|$. Additionally, $\left|X_{1}\right| \leqslant \max \left\{\left|X_{t}^{r}\right|: t \in \mathbb{Z}_{q}\right\},\left|X_{2}\right| \leqslant \max \left\{\left|X_{t}^{c}\right|: t \in \mathbb{Z}_{q}\right\}$, and $\left|X_{3}\right|,\left|X_{4}\right| \leqslant$ $\max \left\{\left|X_{t}^{s}\right|: t \in \mathbb{Z}_{q}\right\}$. Now similarly define

$$
\begin{aligned}
& R_{1}=\left\{v \in H: a=a_{t}+v \text { for some }(a, b, c) \in X\right\}, \\
& R_{2}=\left\{v \in H: b=b_{t+(\ell+1) d}+v \text { for some }(a, b, c) \in X\right\}, \\
& R_{3}=\left\{v \in H: c=a_{t}+b_{t}+v \text { for some }(a, b, c) \in X\right\}, \text { and } \\
& R_{4}=\left\{v \in H: c=a_{t+(\ell+1) d}+b_{t+(\ell+1) d}+v \text { for some }(a, b, c) \in X\right\} .
\end{aligned}
$$

Note that $\left|R_{t}\right|=\left|X_{t}\right|$ for $t \in\{1,2,3,4\}$. Therefore $\left|R_{1} \cup R_{2} \cup R_{3} \cup R_{4}\right|<m$; so there exists some $v \in H \backslash\left(R_{1} \cup R_{2} \cup R_{3} \cup R_{4}\right)$. Then $a_{t}+v \notin X^{r}, b_{t+(\ell+1) d}+v \notin X^{c}, a_{t}+b_{t}+v \notin X_{2 t}^{s}$, and $a_{t+(\ell+1) d}+b_{t+(\ell+1) d}+v \notin X_{2 t+d}^{s}$. Then $P_{t}(v)$ is a $(d, H)$-chain of $C(G)$ with respect to $\mu$ which still contains $(0,0,0)$, whose swap contains $\left(w^{\prime}, z^{\prime}, 0\right)$, and contains fewer cells which are not disjoint from $X$. Proceeding iteratively, we may produce a $(d, H)$-chain of $C(G)$ with respect to $\mu$ which contains $(0,0,0)$, whose swap contains $\left(w^{\prime}, z^{\prime}, 0\right)$, and is row-, column-, and symbol-disjoint from $X$. 


\section{Completing Partial Transversals}

We begin with a proof of Theorem 6 with $k=2$; note that Theorem 1 follows as a special case when the group $G$ is cyclic.

Proof of Theorem 6 with $k=2$. The forward direction is straightforward, so we focus on the reverse direction. Let $n$ be odd and $n \geqslant 3$. Let $P$ be a partial transversal of $C(G)$ and, without loss of generality, we may assume $P=\{(0,0,0),(a, b, a+b)\}$ for some nonzero $a, b \in G$ with $a+b \neq 0$.

If $n$ is prime, then we may assume $G=\mathbb{Z}_{n}$. There exists $v \in \mathbb{Z}_{n}$ so that $a v=b(\bmod n)$; then $\left\{(i, v i, i+v i): i \in \mathbb{Z}_{n}\right\}$ is a completion of $P$. Now assume $n$ is composite and, if $G^{\prime}$ is any Abelian group of odd order $n^{\prime}$ with $3 \leqslant n^{\prime}<n$, then any partial transversal of $C\left(G^{\prime}\right)$ with length 2 is completable.

Let $G$ be an Abelian group of odd order $n=m q$ with $q$ prime, $m \geqslant 3$, and an order $m$ subgroup $H$. Let $\mu: \mathbb{Z}_{q} \rightarrow G / H$ be an isomorphism with $\mu(i)=H_{i}$ for $i \in \mathbb{Z}_{q}$. Let $\alpha, \beta \in \mathbb{Z}_{q}$ such that $a \in H_{\alpha}$ and $b \in H_{\beta}$, and thus $(a, b, a+b) \in C_{\alpha, \beta}(H)$. We consider four cases.

Case 1: Suppose $\alpha=\beta=0$. By the inductive hypothesis, $P$ has a completion $\tau_{0}$ in $C_{0,0}(H)$. For each nonzero $k \in \mathbb{Z}_{q}$, let $\tau_{k}$ be a transversal of $C_{k, k}(H)$. Then $\tau_{0} \cup \tau_{1} \cup \cdots \cup \tau_{q-1}$ is a completion of $P$.

Case 2: Suppose each of $\alpha, \beta$, and $\alpha+\beta$ are nonzero. Again by the inductive hypothesis, $\{(0,0,0),(\alpha, \beta, \alpha+\beta)\}$ has a completion $\tau^{\prime}$ in $C\left(\mathbb{Z}_{q}\right)$. Let $\sigma$ be the permutation of $\mathbb{Z}_{q}$ so that $\tau^{\prime}=\left\{(v, \sigma(v), v+\sigma(v)): v \in \mathbb{Z}_{q}\right\}$. Necessarily $\sigma(0)=0$ and $\sigma(\alpha)=\beta$. For each $v \in \mathbb{Z}_{q}$, let $\tau_{v}$ be a transversal of $C_{v, \sigma(v)}(H)$ so that $\tau_{0}$ and $\tau_{\alpha}$ are completions of $\{(0,0,0)\}$ and $\{(a, b, a+b)\}$ in $C_{0,0}(H)$ and $C_{\alpha, \sigma(\alpha)}(H)$, respectively. Then $\tau_{0} \cup \tau_{1} \cup \cdots \cup \tau_{q-1}$ is a completion of $P$.

Case 3: Suppose $\alpha$ and $\beta$ are nonzero and $\alpha+\beta=0$. Let $c \in H$ so that $2 c=a+b$. Then by Lemma 15 (with $X$ empty), we may define $Q=\left\{\left(x_{i}, y_{i}, x_{i}+y_{i}\right) \in C_{i, i}(H): i \in \mathbb{Z}_{q}\right\}$ as a $(\beta-\alpha, H)$-chain with respect to $\mu$ containing $(c, c, a+b)$ (which then implies $x_{0}=y_{0}=c$ ) and whose swap contains $(a, b, a+b)$. By the inductive hypothesis, let $\tau_{0}$ be a completion of $\{(0,0,0),(c, c, a+b)\}$ in $C_{0,0}(H)$, and for each nonzero $v \in \mathbb{Z}_{q}$, let $\tau_{v}$ be a completion of $\left\{\left(x_{v}, y_{v}, x_{v}+y_{v}\right)\right\}$ in $C_{v, v}(H)$. Then $\left(\left(\tau_{0} \cup \tau_{1} \cup \cdots \cup \tau_{q-1}\right) \backslash Q\right) \cup \sigma(Q)$ is a completion of $P$.

Case 4: Suppose $\alpha=0$ and $\beta \neq 0$. By Lemma 14, let $Q=\left\{\left(x_{i}, y_{i}, x_{i}+y_{i}\right) \in C_{i, i}(H)\right.$ : $\left.i \in \mathbb{Z}_{q}\right\}$ be a $(\beta, H)$-chain of $C(G)$ with respect to $(H, \mu)$ containing $(a, a, 2 a)$ and whose swap contains $(a, b, a+b)$. By the inductive hypothesis, let $\tau_{0}$ be a completion of $\{(0,0,0),(a, a, 2 a)\}$ in $C_{0,0}(H)$, and for each nonzero $v \in \mathbb{Z}_{q}$, let $\tau_{v}$ be a completion of $\left\{\left(x_{v}, y_{v}, x_{v}+y_{v}\right)\right\}$ in $C_{v, v}(H)$. Then $\left(\left(\tau_{0} \cup \tau_{1} \cup \cdots \cup \tau_{q-1}\right) \backslash Q\right) \cup \sigma(Q)$ is a completion of $P$. An identical argument holds if $\alpha \neq 0$ and $\beta=0$ by using transposes. 
The remainder of this section builds an argument for completing partial transversals of $C(G)$ with length 3 , thereby proving the rest of Theorem 6 . In what follows, when a partial transversal $P$ cannot be completed to a transversal in a Latin square $L$, we say $P$ is non-completable in $L$, or simply non-completable.

Observation 16. Note that Theorem 6 does not guarantee that a partial transversal in $C(G)$ with length 3 is completable if $|G|=5$ or 7 . In other words, there may be non-completable partial transversals of $C(G)$ with length 3 if $G$ is isomorphic to $\mathbb{Z}_{5}$ or $\mathbb{Z}_{7}$.

In fact, there are 100 and 294 partial transversals of $C\left(\mathbb{Z}_{5}\right)$ and $C\left(\mathbb{Z}_{7}\right)$ with length 3 , respectively, which are non-completable. These were found by brute-force search and, conveniently, are easily described in the following way. For $p \in\{5,7\}$ and $|G|=p$, the non-completable partial transversals of $C(G)$ with length 3 are of the form

$$
\{(a, b, a+b),(c+a, c+b, 2 c+a+b),(2 c+a,-c+b, c+a+b)\},
$$

where $a, b \in G$, and $c$ is an integer relatively prime to $p$ (any such integer $c$ is sufficient, but note there are only $p-1$ distinct outcomes based on different choices of $c$ ). This partial transversal can be more simply expressed as $\delta_{a, b} \xi_{c}(\{(0,0,0),(1,1,2),(2,-1,1)\})$.

With the above classification, we now present two completion results in particular cases.

Lemma 17. Let $n \in\{25,35,49\}$, $q$ be the smallest prime divisor of $n, n=m q, G$ be an Abelian group of order $n$, and $H$ be the subgroup of $G$ having order $m$. If $\gamma$ is a partial transversal of $C(H)$ with length 3 , then $\gamma$ is completable in $C(G)$.

Proof. Suppose $\tau$ is a completion of $\gamma$ in $C(H)$. Let $\mu: \mathbb{Z}_{q} \rightarrow G / H$ be an isomorphism with $\mu(i)=H_{i}$ for $i \in \mathbb{Z}_{q}$. Then $\tau$ is a transversal of $C_{0,0}(H)$ as well. For each $i \in\{1,2, \ldots, q-1\}$, let $\tau_{i}$ be any transversal of $C_{i, i}(H)$. Then $\tau \cup \tau_{1} \cup \cdots \cup \tau_{q-1}$ is a completion of $\gamma$ in $C(G)$.

Now assume that $\gamma$ is non-completable in $C(H)$, and let $x \in H$ be nonzero. Note that $\langle x\rangle=H$, and if $G$ is cyclic, we let $x=q$. Then, by Observation 16, there exist $a, b \in H$ and an integer $c$ relatively prime to $m$ such that $\gamma=\delta_{a, b} \xi_{c}(P)$, where $P=$ $\{(0,0,0),(x, x, 2 x),(2 x,-x, x)\}$. Observe that $\delta_{a, b} \xi_{c}$ is also an invariant map of $C(G)$, as $a, b \in G$ and $c$ is relatively prime to $n$, except when $m=7$ and $q=c=5$; in this case use $c=-2$. In Figure 4, we give completions of $P$ in all five cases. Therefore, $\gamma$ is completable in $C(G)$ as well.

Lemma 18. Let $n \in\{25,35,49\}$. Let $G$ be an Abelian group of odd order $n=m q$ with $q$ the smallest prime divisor of $n$, and $H$ be an order $m$ subgroup of $G$. Let $\mu: \mathbb{Z}_{q} \rightarrow G / H$ be an isomorphism with $\mu(i)=H_{i}$ for $i \in \mathbb{Z}_{q}$. Let $T_{1}, T_{2} \in C(G)$ such that $(0,0,0), T_{1}$, and $T_{2}$ belong to distinct block diagonals of $C(G)$ with respect to $(H, \mu)$. If $\gamma=\left\{(0,0,0), T_{1}, T_{2}\right\}$ is a partial transversal of $C(G)$, then $\gamma$ is completable in $C(G)$. 


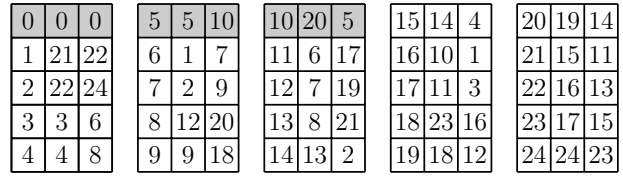

(a)

\begin{tabular}{|c|c|c|c|c|}
\hline \begin{tabular}{|l|l|l|}
0 & 00 & 00 \\
\end{tabular} & \begin{tabular}{|l|l|l|}
10 & 20 & 30 \\
\end{tabular} & \begin{tabular}{|l|l|l|}
20 & 33 & 03 \\
\end{tabular} & \begin{tabular}{|l|l|l|}
30 & 32 & 12 \\
\end{tabular} & \begin{tabular}{|l|l|l|}
40 & 03 & 43 \\
\end{tabular} \\
\hline \begin{tabular}{|l|l|l|}
01 & 01 & 02 \\
\end{tabular} & \begin{tabular}{|l|l|l|}
11 & 10 & 21 \\
\end{tabular} & \begin{tabular}{|l|l|l|}
21 & 21 & 42 \\
\end{tabular} & \begin{tabular}{|l|l|l}
31 & 34 & 10 \\
\end{tabular} & \begin{tabular}{|l|l|l|}
41 & 41 & 32 \\
\end{tabular} \\
\hline \begin{tabular}{|l|l|l|}
02 & 04 & 01 \\
\end{tabular} & \begin{tabular}{|l|l|l|}
12 & 11 & 23 \\
\end{tabular} & \begin{tabular}{|l|l|l|}
22 & 22 & 44 \\
\end{tabular} & \begin{tabular}{|l|l|l|}
32 & 42 & 24 \\
\end{tabular} & \begin{tabular}{|l|l|l|}
42 & 44 & 31 \\
\end{tabular} \\
\hline \begin{tabular}{l|lll}
03 & 13 & 11 \\
\end{tabular} & \begin{tabular}{|l|l|l|l}
13 & 12 & 2 \\
\end{tabular} & \begin{tabular}{|l|l|l|}
23 & 23 & 41 \\
\end{tabular} & \begin{tabular}{|l|l|l|}
33 & 31 & 14 \\
\end{tabular} & \begin{tabular}{|l|l|l}
43 & 02 & 40 \\
\end{tabular} \\
\hline 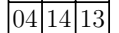 & \begin{tabular}{|l|l|l|}
14 & 24 \\
\end{tabular} & \begin{tabular}{|l|l|l|}
24 & 30 & 04 \\
\end{tabular} & \begin{tabular}{|l|l|l|}
34 & 43 & 22 \\
\end{tabular} & \\
\hline
\end{tabular}

(b)

\begin{tabular}{|c|c|c|c|c|c|}
\hline 0 & \begin{tabular}{|l|l|}
0 & 0 \\
\end{tabular} & \begin{tabular}{l|l|l|l}
7 & 7 & 14 \\
\end{tabular} & \begin{tabular}{l|l|l}
14 & 29 & 8
\end{tabular} & \begin{tabular}{|l|l|l}
21 & 21 & 7 \\
\end{tabular} & \begin{tabular}{|l|l|l|}
28 & 24 & 17 \\
\end{tabular} \\
\hline 1 & \begin{tabular}{l|l}
1 & 2 \\
\end{tabular} & \begin{tabular}{l|l|l|}
8 & 8 & 16 \\
\end{tabular} & \begin{tabular}{l|l|l|}
15 & 15 & 30 \\
\end{tabular} & \begin{tabular}{|l|l|l|l|l|l|}
22 & 22 \\
\end{tabular} & \begin{tabular}{|l|l|l|l}
29 & 19 \\
\end{tabular} \\
\hline 2 & \begin{tabular}{|l|l|}
2 & 4 \\
\end{tabular} & \begin{tabular}{l|l|l}
9 & 14 & 23 \\
\end{tabular} & \begin{tabular}{|l|l|l|}
16 & 16 & 32 \\
\end{tabular} & \begin{tabular}{|l|l|l|}
23 & 4 & 2 \\
\end{tabular} & \begin{tabular}{|l|l|l|}
30 & 25 & 20 \\
\end{tabular} \\
\hline 3 & \begin{tabular}{|l|l|}
3 & 6 \\
\end{tabular} & \begin{tabular}{|l|l|l}
10 & 30 & 5 \\
\end{tabular} & \begin{tabular}{|l|l|l|}
17 & 17 \\
\end{tabular} & \begin{tabular}{|l|l|}
9 & 3 \\
\end{tabular} & \begin{tabular}{|l|l|l|}
31 & 32 & 28 \\
\end{tabular} \\
\hline 4 & \begin{tabular}{|l|l|}
34 & 3 \\
\end{tabular} & \begin{tabular}{|l|l|l|}
11 & 11 & 22 \\
\end{tabular} & \begin{tabular}{|l|l|l|}
18 & 18 & 1 \\
\end{tabular} & \begin{tabular}{|l|l|l|}
25 & 31 & 2 \\
\end{tabular} & \begin{tabular}{|l|l|l}
32 & 28 & 25 \\
\end{tabular} \\
\hline 5 & \begin{tabular}{|l|l|}
5 & 10 \\
\end{tabular} & \begin{tabular}{|l|l|l|}
12 & 12 & 24 \\
\end{tabular} & \begin{tabular}{|l|l|l|l|}
19 & 29 \\
\end{tabular} & \begin{tabular}{|l|l|l}
26 & 27 & 1 \\
\end{tabular} & \begin{tabular}{|l|l|l|}
33 & 33 & 31 \\
\end{tabular} \\
\hline 6 & \begin{tabular}{|l|l|}
6 & 12 \\
\end{tabular} & \begin{tabular}{|l|l|l|}
13 & 13 & 26 \\
\end{tabular} & \begin{tabular}{|l|l|l|}
20 & 26 & 11 \\
\end{tabular} & \begin{tabular}{|l|l|l}
27 & 23 & 1
\end{tabular} & \begin{tabular}{|l|l|l|}
34 & 20 & 19 \\
\end{tabular} \\
\hline
\end{tabular}

(c)

\begin{tabular}{|c|c|c|c|c|c|c|c|c|}
\hline & \begin{tabular}{|l|l|}
0 & 0 \\
\end{tabular} & 7 & \begin{tabular}{|l|l|}
7 & 14 \\
\end{tabular} & \begin{tabular}{|l|l|l|}
14 & 42 & 7 \\
\end{tabular} & \begin{tabular}{|l|l|l|}
21 & 21 & 42 \\
\end{tabular} & \begin{tabular}{|l|l|l|}
28 & 20 \\
\end{tabular} & \begin{tabular}{|l|l|l|}
35 & 35 & 21 \\
\end{tabular} & \begin{tabular}{|l|l|l|}
42 & 34 & 27
\end{tabular} \\
\hline & \begin{tabular}{l|l|}
36 & 37
\end{tabular} & 8 & \begin{tabular}{|l|l|}
43 & 2 \\
\end{tabular} & \begin{tabular}{|l|l|l}
15 & 1 & 16 \\
\end{tabular} & \begin{tabular}{|l|l|l|}
22 & 8 & 30 \\
\end{tabular} & \begin{tabular}{|l|l|l|}
29 & 14 & 43 \\
\end{tabular} & \begin{tabular}{|l|l|l}
36 & 22 & 9 \\
\end{tabular} & \begin{tabular}{|l|l|l}
43 & 28 & 22 \\
\end{tabular} \\
\hline & \begin{tabular}{|l|l|}
37 & 39 \\
\end{tabular} & \begin{tabular}{|l|}
9 \\
\end{tabular} & \begin{tabular}{l|l}
44 & 4 \\
\end{tabular} & \begin{tabular}{|l|l|l}
16 & 2 & 18 \\
\end{tabular} & \begin{tabular}{|l|l|l|}
23 & 9 & 32 \\
\end{tabular} & \begin{tabular}{|l|l|l|}
30 & 15 & 45 \\
\end{tabular} & \begin{tabular}{|l|l|l|}
37 & 23 & 11 \\
\end{tabular} & \begin{tabular}{|l|l|l|}
44 & 29 & 24 \\
\end{tabular} \\
\hline & \begin{tabular}{l|l|}
38 & 41
\end{tabular} & $10[4$ & \begin{tabular}{l|l}
45 & 6
\end{tabular} & \begin{tabular}{|l|l|l}
17 & 3 & 20 \\
\end{tabular} & \begin{tabular}{|l|l|l|}
24 & 10 & 34 \\
\end{tabular} & \begin{tabular}{|l|l|l|}
31 & 16 & 47 \\
\end{tabular} & \begin{tabular}{|l|l|}
24 & 13 \\
\end{tabular} & \begin{tabular}{|l|l|l|}
45 & 30 & 26 \\
\end{tabular} \\
\hline & \begin{tabular}{l|l|}
31 & 35 \\
\end{tabular} & \begin{tabular}{|l|l|}
11 & 1 \\
\end{tabular} & \begin{tabular}{l|l|}
17 & 28 \\
\end{tabular} & \begin{tabular}{l|l|l|}
18 & 46 & 15 \\
\end{tabular} & \begin{tabular}{|l|l|l|}
25 & 11 & 36 \\
\end{tabular} & \begin{tabular}{|l|l|l}
32 & 25 & 8 \\
\end{tabular} & \begin{tabular}{|l|l|l|}
39 & 39 & 29 \\
\end{tabular} & \begin{tabular}{|l|l|}
46 & 4 \\
\end{tabular} \\
\hline & \begin{tabular}{|l|l|}
47 & 3 \\
\end{tabular} & \begin{tabular}{|l|l|}
12 \\
\end{tabular} & \begin{tabular}{|l|l|}
5 & 17 \\
\end{tabular} & \begin{tabular}{|l|l|l|}
19 & 12 & 31 \\
\end{tabular} & \begin{tabular}{|l|l|l|}
26 & 18 & 44 \\
\end{tabular} & \begin{tabular}{|l|l|l|}
33 & 26 & 10 \\
\end{tabular} & \begin{tabular}{|l|l|l|}
40 & 32 & 23 \\
\end{tabular} & \begin{tabular}{|l|l|l|}
47 & 40 & 38 \\
\end{tabular} \\
\hline & $\begin{array}{ll}485 \\
5\end{array}$ & & \begin{tabular}{|l|l|}
6 & 19 \\
\end{tabular} & \begin{tabular}{|l|l|l|}
20 & 13 & 33 \\
\end{tabular} & \begin{tabular}{|l|l|l|}
27 & 19 & 46 \\
\end{tabular} & \begin{tabular}{|l|l|l|}
34 & 27 & 12 \\
\end{tabular} & \begin{tabular}{|l|l|l|}
41 & 33 & 25 \\
\end{tabular} & \begin{tabular}{|l|l|l|}
48 & 41 & 40 \\
\end{tabular} \\
\hline
\end{tabular}

(d)

\begin{tabular}{|c|c|c|c|c|c|c|}
\hline \begin{tabular}{|l|l|}
00 & 00 \\
\end{tabular} & \begin{tabular}{|l|l|l|l}
10 & 11 \\
\end{tabular} & \begin{tabular}{|l|l|l|l}
20 & 20 \\
\end{tabular} & \begin{tabular}{|l|l|l|}
30 & 30 & 60 \\
\end{tabular} & \begin{tabular}{|l|l|l|}
40 & 56 & 26 \\
\end{tabular} & \begin{tabular}{|l|l|l|}
50 & 52 & 32 \\
\end{tabular} & \begin{tabular}{|l|l|l|}
60 & 66 & 56 \\
\end{tabular} \\
\hline \begin{tabular}{|l|l|}
01 & 02 \\
\end{tabular} & \begin{tabular}{|l|l|l}
11 & 12 & 23 \\
\end{tabular} & \begin{tabular}{|l|l|l}
21 & 21 & 42 \\
\end{tabular} & \begin{tabular}{|l|l|l|}
31 & 31 & 62 \\
\end{tabular} & \begin{tabular}{|l|l|l}
41 & 43 & 14 \\
\end{tabular} & \begin{tabular}{|l|l|l|}
51 & 53 & 34 \\
\end{tabular} & \begin{tabular}{|l|l|l|}
61 & 60 & 51 \\
\end{tabular} \\
\hline \begin{tabular}{|l|l|}
06 & 01 \\
\end{tabular} & \begin{tabular}{|l|l|l|}
12 & 13 & 25 \\
\end{tabular} & \begin{tabular}{|l|l|l}
22 & 22 & 44 \\
\end{tabular} & \begin{tabular}{|l|l|l|}
32 & 32 & 64 \\
\end{tabular} & \begin{tabular}{|l|l|l|}
42 & 46 & 11 \\
\end{tabular} & \begin{tabular}{|l|l|l|l|}
52 & 54 & 36 \\
\end{tabular} & \begin{tabular}{|l|l|l|}
62 & 62 & 54 \\
\end{tabular} \\
\hline \begin{tabular}{|l|l|}
03 & 06 \\
\end{tabular} & \begin{tabular}{|l|l|l|}
13 & 14 & 20 \\
\end{tabular} & \begin{tabular}{|l|l|l}
23 & 23 & 46 \\
\end{tabular} & \begin{tabular}{|l|l|l|}
33 & 33 & 66 \\
\end{tabular} & \begin{tabular}{|l|l|l}
43 & 42 & 15 \\
\end{tabular} & \begin{tabular}{|l|l|l|}
53 & 55 & 31 \\
\end{tabular} & \begin{tabular}{|l|l|l}
63 & 02 & 65 \\
\end{tabular} \\
\hline \begin{tabular}{|l|l|}
16 & 13 \\
\end{tabular} & \begin{tabular}{|l|l|l|}
14 & 15 & 22 \\
\end{tabular} & \begin{tabular}{|l|l|l|}
24 & 24 & 41 \\
\end{tabular} & \begin{tabular}{|l|l|l|}
34 & 34 & 61 \\
\end{tabular} & \begin{tabular}{|l|l|l|}
44 & 50 & 24 \\
\end{tabular} & \begin{tabular}{|l|l|l|}
54 & 61 & 45 \\
\end{tabular} & \begin{tabular}{|l|l|l|}
64 & 63 & 50
\end{tabular} \\
\hline $\begin{array}{lll}05 & 03 \\
\end{array}$ & \begin{tabular}{|l|l|l|}
15 & 25 & 33 \\
\end{tabular} & \begin{tabular}{|l|l|l}
25 & 35 & 53 \\
\end{tabular} & \begin{tabular}{|l|l|l|}
35 & 40 & 05 \\
\end{tabular} & \begin{tabular}{|l|l|l}
45 & 44 & 12 \\
\end{tabular} & \begin{tabular}{|l|l|l}
55 & 65 & 43 \\
\end{tabular} & \begin{tabular}{|l|l|l|}
65 & 64 & 52 \\
\end{tabular} \\
\hline \begin{tabular}{|l|l|}
10 & 16 \\
\end{tabular} & \begin{tabular}{|l|l|l|}
16 & 35 \\
\end{tabular} & \begin{tabular}{|l|l|l|}
26 & 36 & 55 \\
\end{tabular} & \begin{tabular}{|l|l|l|}
36 & 45 & 04 \\
\end{tabular} & \begin{tabular}{|l|l|l}
46 & 41 & 10 \\
\end{tabular} & \begin{tabular}{|l|l|l|}
56 & 51 & 30 \\
\end{tabular} & \begin{tabular}{|l|l|l|}
66 & 04 & 63 \\
\end{tabular} \\
\hline
\end{tabular}

(e)

Figure 4: Completions of $P$ as described in Lemma 17 for (a) $C\left(\mathbb{Z}_{25}\right)$, (b) $C\left(\mathbb{Z}_{5} \times \mathbb{Z}_{5}\right)$, (c) $C\left(\mathbb{Z}_{35}\right)$, (d) $C\left(\mathbb{Z}_{49}\right)$, and (e) $C\left(\mathbb{Z}_{7} \times \mathbb{Z}_{7}\right)$. For each noncyclic group $\mathbb{Z}_{p} \times \mathbb{Z}_{p}$, we fix $y \in G \backslash H$; note that $\langle x, y\rangle=G$ and each element of $G$ is representable as $a x+b y$ for some $a, b \in \mathbb{Z}_{p}$, and we use $a b$ to represent the element $a x+b y$ in the charts above.

Computer Proof. Despite our best efforts, the only way we found to justify this is through a brute-force computer search. Let $d$ and $e$ be the indices of the block diagonals with respect to $(H, \mu)$ containing $T_{1}$ and $T_{2}$, respectively. Then perform the following procedure:

1. Randomly construct an SDR of $G / H$ including the symbol of $T_{1}$, then construct a $(d, H)$-chain $D$ of $C(G)$ with respect to $(H, \mu)$ using the SDR and the row of $T_{1}$. Note that $T_{1} \in \sigma(D)$. If the chain does not avoid $(0,0,0)$, then restart the process.

2. Randomly construct an SDR of $G / H$ including the symbol of $T_{2}$, then construct an $(e, H)$-chain $E$ of $C(G)$ with respect to $(H, \mu)$ using the SDR and the row of $T_{2}$. Note that $T_{2} \in \sigma(E)$. If the chain does not avoid $D$ or does not avoid $(0,0,0)$, then restart the process.

3. Check that the partial transversal $P=(D \cup E \cup\{(0,0,0)\}) \cap C(H)$ with length 3 is completable in $C(H)$. For this, we simply check that $P$ is not a non-completable partial transversal of $C(H)$ as classified in Observation 16. If $P$ is non-completable in $C(H)$, then restart the process.

If the process succeeds, there exists a completion $\tau_{0}$ of $P$ in $C(H)$ and for each nonzero $i \in \mathbb{Z}_{q}$, there exists a completion $\tau_{i}$ of $(D \cup E) \cap C_{i, i}(H)$ in $C_{i, i}(H)$, the latter of which follows from Theorem 1. Then $\left(\tau_{0} \cup \tau_{1} \cup \cdots \cup \tau_{q-1} \cup \sigma(D) \cup \sigma(E)\right) \backslash(D \cup E)$ is a completion of $\gamma$ in $C(G)$. We provide python code for this procedure, as well as some sample outputs 
here. Note that this routine successfully completed each possible $\gamma$, with very infrequent restarts. This leads us to speculate there are many chains which may be used to produce a completion, while very few would cause a conflict.

We now present a lemma highlighting how choosing the subgroup $H$ wisely can affect the location of cells in coset blocks of $C(G)$. Afterward, we describe another brute-force method for resolving another small case, and then conclude with the remaining proof of Theorem 6 .

Lemma 19. Let $p$ be prime, $G=\mathbb{Z}_{p} \times \mathbb{Z}_{p}$, and $T \in C(G)$. Then there exists a subgroup $H$ of $G$ with order $p$ such that for all $\mu: \mathbb{Z}_{p} \rightarrow G / H, T$ belongs to the main block diagonal of $C(G)$ with respect to $(H, \mu)$.

Proof. Let $x_{1}, x_{2}, y_{1}, y_{2} \in \mathbb{Z}_{p}$ so that $a=\left(x_{1}, y_{1}\right), b=\left(x_{2}, y_{2}\right)$, and $T=(a, b, a+b)$. If $x_{1}=x_{2}$, then $b-a \in\langle(0,1)\rangle$. Otherwise, $b-a \in\langle(1, \alpha)\rangle$, where $\alpha=\left(x_{2}-x_{1}\right)^{-1}\left(y_{2}-y_{1}\right)$. Hence there exists a subgroup $H$ of $G$ for which $b-a \in H$. So $a$ and $b$ belong to the same coset (say $H_{i}$ ) of $H$, and hence $T \in C_{i, i}(H)$.

Observation 20. The following is a naïve brute-force algorithm to determine which partial transversals of $C(G)$ with length 3 are completable.

- Find the set $\mathcal{P}$ of all partial transversals of $C(G)$ with length 3 containing $(0,0,0)$.

- Find the set $\Pi$ of all permutations $\pi$ of $G$ for which $G=\{i+\pi(i): i \in G\}$ and $\pi(0)=0$. Observe there is a bijection $\phi: \Pi \rightarrow \mathcal{T}$, where $\mathcal{T}$ is the set containing all transversals of $C(G)$ containing $(0,0,0)$, given by $\phi(\pi)=\{(i, \pi(i), i+\pi(i)): i \in G\}$.

- For each $\pi \in \Pi$, let $\mathcal{P}_{\pi}$ be the set of $\left(\begin{array}{c}n-1 \\ 2\end{array}\right)$ partial transversals in $\mathcal{P}$ for which $\phi(\pi)$ is a completion.

- Compare $\mathcal{P}$ to $\cup_{\pi} P_{\pi}$, with the union taken over each $\pi \in \Pi$. If $\mathcal{P}=\cup_{\pi} P_{\pi}$, then all partial transversals of $C(G)$ with length 3 are completable; otherwise $\mathcal{P} \backslash\left(\cup_{\pi} P_{\pi}\right)$ is the set of all non-completable partial transversals of $C(G)$ which contain $(0,0,0)$.

This algorithm quickly becomes infeasible as $|G|$ increases. However, using this technique we confirmed all partial transversals in $C\left(\mathbb{Z}_{9}\right)$ and $C\left(\mathbb{Z}_{3} \times \mathbb{Z}_{3}\right)$ with length 3 are completable; note the former of these two results also follows from Theorem 2. Furthermore, we used this technique to classify the non-completable partial transversals of $C\left(\mathbb{Z}_{5}\right)$ and $C\left(\mathbb{Z}_{7}\right)$ as given in Observation 16. We also provide python code for this procedure here.

Now we conclude with the remaining argument for Theorem 6 .

Proof of Theorem 6 with $k=3$. If $n=3$ the result is trivial; if $n \geqslant 11$ is prime, then the result follows from Theorem 5 ; and if $n=9$ the result follows from Observation 20 .

Now, assume $n \geqslant 15$ is composite, and if $G^{\prime}$ is any Abelian group of odd order $n^{\prime}$ with $9 \leqslant n^{\prime}<n$, then any partial transversal of $C\left(G^{\prime}\right)$ with length 3 is completable in $C\left(G^{\prime}\right)$. 
Let $n=m q$ with $q$ the smallest prime dividing $n$. Then $m \geqslant 5$, and let $H$ be an order $m$ subgroup of $G$. Let $\mu: \mathbb{Z}_{q} \rightarrow G / H$ be an isomorphism with $\mu(i)=H_{i}$ for $i \in \mathbb{Z}_{q}$.

It is sufficient to show any partial transversal $\gamma$ of $C(G)$ with length 3 containing $(0,0,0)$ is completable. Let $\gamma=\left\{T_{0}, T_{1}, T_{2}\right\}$, where $T_{0}=(0,0,0), T_{1}=\left(a_{1}, b_{1}, a_{1}+b_{1}\right)$ and $T_{2}=\left(a_{2}, b_{2}, a_{2}+b_{2}\right)$. Suppose that $T_{1} \in C_{\alpha_{1}, \beta_{1}}(H)$ and $T_{2} \in C_{\alpha_{2}, \beta_{2}}(H)$.

First, suppose $\alpha_{1}=\alpha_{2}=\beta_{1}=\beta_{2}=0$; hence $\gamma \subseteq C(H)$. If $m \geqslant 9$, then by our inductive hypothesis, $\gamma$ is completable to a transversal $\tau$ of $C(H)$. For each nonzero $k \in \mathbb{Z}_{q}$, let $\tau_{k}$ be a transversal of $C_{k, k}(H)$. Then $\tau \cup \tau_{1} \cdots \cup \tau_{q-1}$ is a completion of $\gamma$ in $C(G)$. If $m<9$, then necessarily $(m, q) \in\{(5,3),(5,5),(7,3),(7,5),(7,7)\}$. If $(m, q)=(5,3)$ or $(7,3)$, then $\gamma$ has a completion in $C(G)$ by Theorem 2. Otherwise, $\gamma$ has a completion in $C(G)$ by Lemma 17.

Next, suppose two cells belong to the same coset block; without loss of generality, suppose $T_{1} \in C(H)$ and $T_{2} \notin C(H)$. Since $m \geqslant 3$, there exists a completion $\tau$ of $\left\{T_{0}, T_{1}\right\}$ in $C(H)$. We now consider three subcases which are similar to the proof of Theorem 6 when $k=2$.

Case 1a: Suppose $\alpha_{2}, \beta_{2}$, and $\alpha_{2}+\beta_{2}$ are nonzero. Since $q \geqslant 3,\left\{(0,0,0),\left(\alpha_{2}, \beta_{2}, \alpha_{2}+\beta_{2}\right)\right\}$ has a completion $\tau^{\prime}$ in $C\left(\mathbb{Z}_{q}\right)$; let $\sigma$ be a permutation on $\mathbb{Z}_{q}$ so that $\tau^{\prime}=\{(v, \sigma(v), v+\sigma(v))$ : $\left.v \in \mathbb{Z}_{q}\right\}$. Necessarily $\sigma(0)=0$ and $\sigma\left(\alpha_{2}\right)=\beta_{2}$. Let $\tau_{\alpha_{2}}$ be a completion of $\left\{T_{2}\right\}$ in $C_{\alpha_{2}, \beta_{2}}(H)$, and for each nonzero $i \in \mathbb{Z}_{q}$ with $i \neq \alpha_{2}$, let $\tau_{i}$ be a transversal of $C_{i, \sigma(i)}(H)$. Then $\tau \cup \tau_{1} \cup \cdots \cup \tau_{q-1}$ is a completion of $\gamma$ in $C(G)$.

Case 1b: Suppose $\alpha_{2}$ and $\beta_{2}$ are nonzero but $\alpha_{2}+\beta_{2}=0$; hence $a_{2}+b_{2} \in H$. Let $T^{\prime}$ be the cell in $\tau$ whose symbol is $a_{2}+b_{2}$. By Lemma 15 (with $X=\varnothing$ ), let $P$ be a $\left(\beta_{2}-\alpha_{2}, H\right.$ )-chain with respect to $\mu$ containing $T^{\prime}$ and whose swap contains $T_{2}$. For each nonzero $i \in \mathbb{Z}_{q}$, let $\tau_{i}$ be a completion of $P \cap C_{i, i}(H)$ in $C_{i, i}(H)$. Then $\left(\left(\tau \cup \tau_{1} \cup \cdots \cup \tau_{q-1}\right) \backslash P\right) \cup \sigma(P)$ is a completion of $\gamma$ in $C(G)$.

Case 1c: Suppose $\alpha_{2}=0$ and $\beta_{2}$ is nonzero. Let $T^{\prime}$ be the cell in $\tau$ whose row is $a_{2}$. By proceeding identically to the argument used in Case $1 \mathrm{~b}$, we may again produce a completion of $\gamma$ in $C(G)$. An identical argument holds if $\alpha_{2} \neq 0$ and $\beta_{2}=0$ by using transposes.

For the remaining cases, we may assume no two triples in $\gamma$ belong to the same coset block. We separate these instances into subcases based on how many cells of $\gamma$ share a block diagonal.

Case 2a: Suppose all three triples lie on the same block diagonal; thus $\alpha_{1}=\beta_{1}$ and $\alpha_{2}=\beta_{2}$. Let $\tau_{0}, \tau_{\alpha_{1}}$ and $\tau_{\alpha_{2}}$ be completions of $\left\{T_{0}\right\},\left\{T_{1}\right\}$, and $\left\{T_{2}\right\}$ in $C(H), C_{\alpha_{1}, \alpha_{1}}(H)$, and $C_{\alpha_{2}, \alpha_{2}}(H)$, respectively, and for each $i \in \mathbb{Z}_{q}$ where $i \neq 0, \alpha_{1}, \alpha_{2}$, let $\tau_{i}$ be a transversal of $C_{i, i}(H)$. Then $\tau_{0} \cup \tau_{1} \cup \cdots \cup \tau_{q-1}$ is a completion of $\gamma$ in $C(G)$.

Case 2b: Suppose two of the three triples lie on the same block diagonal. Without loss of generality, we may assume $\alpha_{1}=\beta_{1}$ and $\alpha_{2} \neq \beta_{2}$. Let $\lambda_{2} \in \mathbb{Z}_{q}$ so that $2 \lambda_{2}=\alpha_{2}+\beta_{2}$. Let $X=\left\{T_{0}, T_{1}\right\}$. Let $T^{\prime} \in C_{\lambda_{2}, \lambda_{2}}(H)$ whose symbol is $a_{2}+b_{2}$ and is row- and column-disjoint 
from $X$; this is possible since $m \geqslant 3$. Since $m \geqslant 5>2|X|$, by Lemma 15 there exists a $\left(\beta_{2}-\alpha_{2}, H\right)$-chain $P$ with respect to $\mu$ which is row-, column-, and symbol-disjoint from $X$, $T^{\prime} \in P$, and $T_{2} \in \sigma(P)$. Note that $(X \cup P) \cap C_{i, i}(H)$ is a partial transversal of $C_{i, i}(H)$ with length at most 2 ; hence for each $i \in \mathbb{Z}_{q}$, there exists a completion $\tau_{i}$ of $(X \cup P) \cap C_{i, i}(H)$ in $C_{i, i}(H)$. Then $\left(\left(\tau_{0} \cup \tau_{1} \cup \cdots \cup \tau_{q-1}\right) \backslash P\right) \cup \sigma(P)$ is a completion of $\gamma$ in $C(G)$.

Case 2c: Suppose all three triples lie on different block diagonals; that is $\beta_{1}-\alpha_{1}$ and $\beta_{2}-\alpha_{2}$ are distinct and nonzero. If $m \leqslant 7$, we have that $(m, q)=\{(5,3),(7,3),(5,5),(7,5),(7,7)\}$. If $(m, q) \in\{(5,3),(7,3)\}$, then $G$ is cyclic and thus $\gamma$ is completable in $C(G)$ by Theorem 2 . If $(m, q) \in\{(5,5),(7,5),(7,7)\}$ and $G$ is cyclic, then $\gamma$ is completable in $C(G)$ by Lemma 18 . Otherwise, $(m, q) \in\{(5,5),(7,7)\}$ and $G$ is not cyclic, so by a different choice for $H$, we may assume $\gamma$ has two cells contained in the main block diagonal by Lemma 19; hence these instances are handled in earlier cases.

Now suppose $m \geqslant 9$. For each $i \in\{1,2\}$, let $\lambda_{i} \in \mathbb{Z}_{q}$ so that $2 \lambda_{i}=\alpha_{i}+\beta_{i}$. Since $m \geqslant 5$, there exists a triple $T_{1}^{\prime} \in C_{\lambda_{1}, \lambda_{1}}(H)$ which is row- and column-disjoint from $\left\{T_{0}, T_{2}\right\}$ and whose symbol is $a_{1}+b_{1}$. Since $m>2\left|\left\{T_{0}, T_{2}\right\}\right|$, there exists a $\left(\beta_{1}-\alpha_{1}, H\right)$-chain $P_{1}$ with respect to $\mu$ which is row-, column-, and symbol-disjoint from $\left\{T_{0}, T_{2}\right\}, T_{1}^{\prime} \in P_{1}$, and $T_{1} \in \sigma\left(P_{1}\right)$. Let $X=\left\{T_{0}\right\} \cup P_{1}$. First observe that since $m \geqslant 3$, there exists $T_{2}^{\prime} \in C_{\lambda_{2}, \lambda_{2}}(H)$ which is row- and column-disjoint from $X$ and whose symbol is $a_{2}+b_{2}$. Furthermore, observe that

$\max \left\{\left|X_{i}^{r}\right|: i \in \mathbb{Z}_{q}\right\}+\max \left\{\left|X_{i}^{c}\right|: i \in \mathbb{Z}_{q}\right\}+2 \cdot \max \left\{\left|X_{i}^{s}\right|: i \in \mathbb{Z}_{q}\right\}=\left|X_{0}^{r}\right|+\left|X_{0}^{c}\right|+2\left|X_{0}^{s}\right|=8$.

Since $m \geqslant 9$, there exists a $\left(\beta_{2}-\alpha_{2}, H\right)$-chain $P_{2}$ with respect to $\mu$ which is row-, column-, and symbol-disjoint from $X, T_{2}^{\prime} \in P_{2}$, and $T_{2} \in \sigma\left(P_{2}\right)$. Observe that $\left(\left\{T_{0}\right\} \cup P_{1} \cup P_{2}\right) \cap C(H)$ has a completion $\tau$ in $C(H)$ by the inductive hypothesis. Furthermore, for each nonzero $i \in \mathbb{Z}_{q},\left(P_{1} \cup P_{2}\right) \cap C_{i, i}(H)$ is a partial transversal of $C_{i, i}(H)$ with length 2 with a completion $\tau_{i}$ in $C_{i, i}(H)$ by the proof of Theorem 6 with $k=2$. Therefore $\gamma$ has a completion $\left(\left(\tau \cup \tau_{1} \cup \cdots \cup \tau_{q-1}\right) \backslash\left(P_{1} \cup P_{2}\right)\right) \cup\left(\sigma\left(P_{1}\right) \cup \sigma\left(P_{2}\right)\right)$ in $C(G)$.

\section{Concluding Remarks}

In this paper, we considered partial transversals in Cayley tables of Abelian groups of odd order, and we achieved our result through an iterative approach which may appear to only leverage that the group is solvable. However, in constructing chains, we rely on the group being Abelian. So at this time, we believe a novel method may be required to extend the result to all odd-order groups by leveraging the Feit-Thompson Theorem.

Also, while Cayley tables of cyclic groups have transversals if and only if the order is odd, the same cannot be said for arbitrary Abelian groups of finite order. Let $G$ be a finite Abelian group. A necessary condition for $C(G)$ to contain a transversal is that the sum of all elements of $G$ is 0 , which is true for odd order cyclic groups, whereas such a sum is $|G| / 2$ for even-order cyclic groups. However, the necessary condition is sufficient for the 
existence of transversals in $C(G)$, regardless of the parity of $|G|$, through an application of a result discovered by Marshall Hall Jr. [5]. Observe that if $|G|$ is even, then the sum of all elements of $G$ is 0 if and only if $\mathbb{Z}_{2} \times \mathbb{Z}_{2}$ is a subgroup of $G$. This raises the following general question.

Question 21. Let $G$ be an Abelian group for which all elements of $G$ sum to zero. Given a positive integer $k$, is there a threshold $d_{k}$ such that if $|G| \geqslant d_{k}$, then all partial transversals of length $k$ in $C(G)$ are completable?

Another result used in this work was the classification of non-completable partial transversals of length 3. Given they have such a nice structure, this could imply a similar structure for non-completable partial transversals in $C(G)$ with length $k$ as $|G|$ approaches $3 k-1$ from below. While this looks interesting, this seems to be a much harder classification problem.

Finally, we want to thank the anonymous referees of our paper who helped with clarifying exposition and pushed us to make our results stronger.

\section{References}

[1] Nicholas J. Cavenagh, Carlo Hämäläinen, and Adrian M. Nelson. On completing three cyclically generated transversals to a Latin square. Finite Fields Appl., 15:294-303, 2009.

[2] Sean Eberhard, Freddie Manners, and Rudi Mrazović. Additive triples of bijections, or the toroidal semiqueens problem. J. Eur. Math. Soc., 21:441-463, 2019.

[3] Martin Grüttmüller. Completing partial Latin squares with two cyclically generated prescribed diagonals. J. Combin. Theory Ser. A, 103:349-362, 2003.

[4] Martin Grüttmüller. Completing partial Latin squares with prescribed diagonals. Discrete Appl. Math., 138:89-97, 2004.

[5] Marshall Hall, Jr. A combinatorial problem on abelian groups. Proc. Amer. Math. Soc., 3:584-587, 1952.

[6] Ian M. Wanless. Diagonally cyclic Latin squares. European J. Combin., 25:393-413, 2004.

[7] Ian M. Wanless and Bridget S. Webb. The existence of Latin squares without orthogonal mates. Des. Codes Cryptogr., 40:131-135, 2006. 